\title{
Development of a Commodity Location Determining Method for Manual Picking Efficiency in an Unautomated Warehouse
}

\author{
Tsuyoshi Takahashi ${ }^{\mathrm{a},}$, Makoto Nishida ${ }^{\mathrm{a}}$, Yoichi Kageyama ${ }^{\mathrm{a}}$, and Hachiro Endo ${ }^{\mathrm{b}}$ \\ ${ }^{a}$ Akita University, 1-1 Tegata Gakuen-machi, Akita City, Akita 010-8502, Japan \\ ${ }^{\mathrm{b}}$ Logizard Co. Ltd., 3-3-6 Ningyo-cho, Chuo, Tokyo 103-0013, Japan \\ *Corresponding Author: tsuyoshi@ie.akita-u.ac.jp
}

\begin{abstract}
It has recently been shown that a reduction in distribution cost is needed to both ensure lower prices and a higher quality of products. The expansion of the Internet mail order system requires warehouses to handle many types of commodities and small quantities of these. Picking operations in the warehouses are dependent on individuals who can respond flexibly. Therefore, shipment work has become a labor-intensive operation. In particular, walking time for commodity picking accounts for over half of the total shipping time.

In this study, we propose a commodity location method to reduce the manual picking travel distance for the warehouse that handles many types and small quantities of commodities. We focus on the order quantity for using $\mathrm{ABC}$ analysis to determine the commodity location. Our experimental results suggest that the proposed method can reduce the traveling time for manual picking.
\end{abstract}

Keywords: Warehouse, Total picking, Commodity location

\section{Introduction}

Presently, many companies try to reduce storage and distribution costs related to their warehouses ${ }^{(1-4)}$. In the past, the main role of the warehouse was to store many supplies ${ }^{(2,}$ 6). However, warehouses have now changed to physical distribution centers that distribute several products with efficiency. Many parts of a warehouse's functions have been automated and work efficiency improved using information technology.

It has been identified that order picking is last activity remaining to be automated in almost all medium or small-sized warehouses ${ }^{(1-3)}$. Automation and robots are rarely used for picking in such warehouses. Therefore, order picking is the most labor-intensive and costly activity in those warehouses. The cost of order picking generally accounts for as much as $54 \%$ of the total shipping operating expenses ${ }^{(2)}$. The picking operation can be divided into multiple of work processes, including "travel," "picking commodities," and "confirming commodities." According to an operation time analysis, traveling time occupies over half of the working time (approximately 52\%) in order picking $^{(1,2)}$. To reduce the traveling time, the picker needs to memorize a commodity location, and choose the shorter route for picking all orders. Furthermore, the layout of a warehouse can change with new items or replacement of major seasonal items. Therefore, it is difficult to satisfy these requirements even for expert workers.

Fig. 1 shows the outline of the picking support system. One of the methods for improving picking work efficiency is to locate the commodities' in appropriate racks ${ }^{(1,5)}$. In this study, we develop the commodity location method for the picking support system used in medium or small-sized warehouses. We tested the method with actual order data for seven months, and evaluated the usefulness of our proposed location method.

The remainder of this paper is organized as follows. In Section 2, we describe the details of the warehouse model used in this study. In Section 3, the details of the proposed

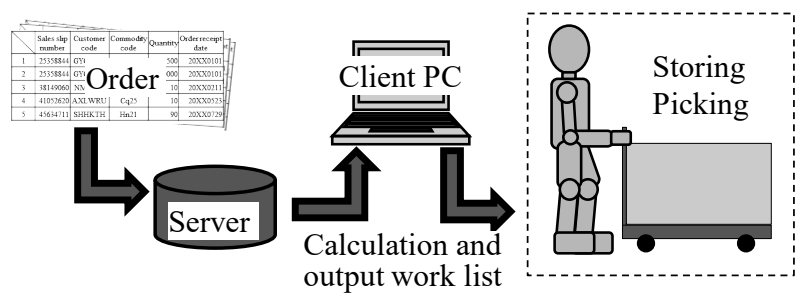

Fig. 1. Outline of a picking support system. 
method using $\mathrm{ABC}$ analysis ${ }^{(2,6)}$ are described. In Section 4, we describe the experiment and evaluation of the proposed method. The experiment aims to investigate the usefulness of the proposed method. We compare the travel distance between the proposed method and the commodity location (original location) based on general warehouse knowledge ${ }^{(2,3)}$. Section 5 discusses the conclusions of this study.

\section{Warehouse and Picking Model}

\subsection{Layout Design}

The target of consideration of this study is the medium or small-sized warehouse. The target warehouses have less automated facilities such as a robotized picking system and therefore handle various items and small lot sizes. Therefore, almost all the work is labor-intensive, particularly in a manual picking operation. Fig. 2 shows the storage rack (shelf) layout used in this study. It is a typical layout for a medium or small-sized warehouse. Two storage zones are situated opposite to each other with the aisle in the center. Both zones have the same layout. The number of shelves is the minimum value calculated from the number of varieties of items. The width of all aisles is wide enough for two handcarts to pass each other. The depot (shipping place) is placed just outside the entrance door. In this model, each rack stores one type of item, and the items can be replenished at any time.

\subsection{Picking Model}

In this study, we assumed as a work model that one picker works at a single site exclusively. They pick order items using the total picking method that involves collecting items together from multiple customer orders. This model uses a general type handcart for the transportation of the shipping commodities. Therefore, carrying means are determined as the volume of a container that can be mounted onto the handcart. If the amount of the order commodity is larger than the container volume, the picker must return to the depot and unload the picked commodities.

\section{Location Determining Method}

\subsection{Outline of Location Determining Method}

The shipping quantity of each commodity is generally different due to various factors such as the consumption degree or popularity of each commodity. The shipping

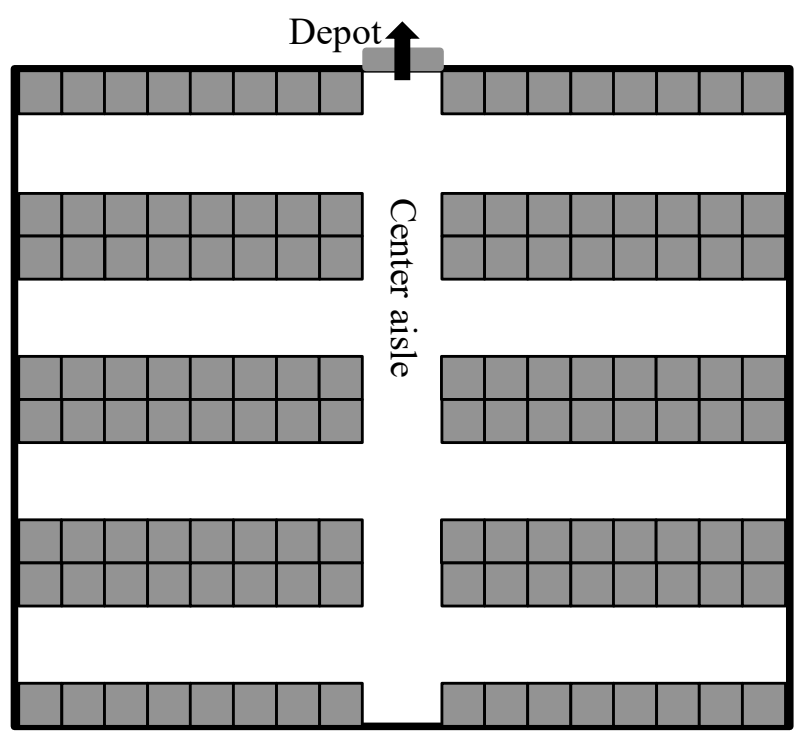

Fig. 2. Layout design image (single site).

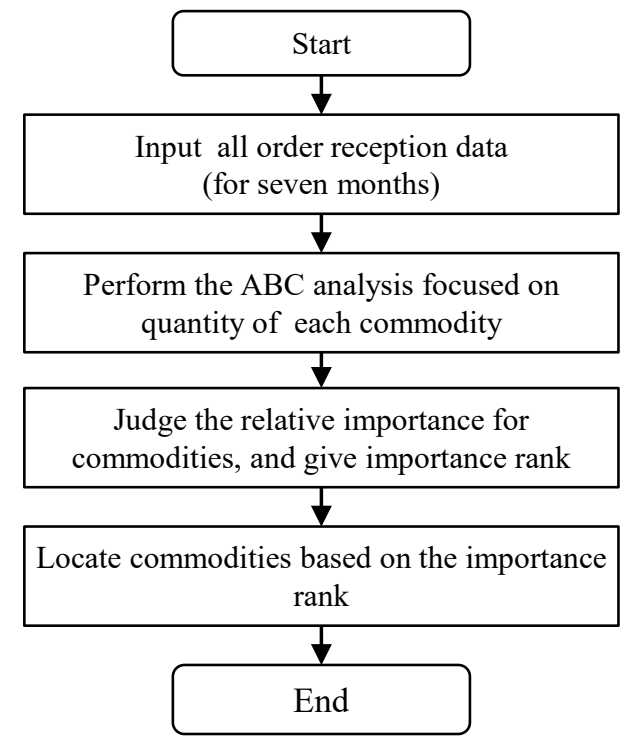

Fig. 3. Outline of proposed method.

quantity and order frequency of each commodity significantly influence the travelling distance of the picker. From this factor, it is thought that locating the commodities based on shipping quantity priority is useful for shortening such travelling distance. Therefore, we propose a location determining method focused on the shipping quantities. The proposed method locates the commodities near the entrance in the order of the shipping quantity in all orders for seven months. Fig. 3 shows the outline of the proposed location determining method using $\mathrm{ABC}$ analysis ${ }^{(2,6)}$. The proposed method consists of four steps. First, input all of the order reception data for seven months. Second, perform ABC analysis focused on the quantity of each commodity. Third, judge the relative importance of each commodity, and 
assign an importance rank. Finally, locate commodities based on the importance rank judged by a Pareto chart.

\subsection{Data Used}

Table 1 lists an example of order reception data (raw data). The data have five basic records. Orders from the same customer have the same customer code. In this study, we use three types of records: commodity code, shipment quantity, and order receipt date. We convert the order reception data to a total picking slip in four steps. First, read the raw data and divide the original receipt orders by each different order receipt date. Second, divide orders for one day equally into three work units based on our field survey at the target type warehouse. Third, find the total orders of each unit by commodity code. Finally, add one more record, the "commodity location number" for every commodity sequentially. The commodity location number is the same as the rack number such as 1 to $n$ for storing one type of commodity by one rack. Then, a total of three picking slips are generated for a day.

\subsection{ABC Analysis for All Orders}

For determining the commodity location adaptively, we use $\mathrm{ABC}$ analysis. Fig. 4 shows the flow of $\mathrm{ABC}$ analysis in our proposed method. $\mathrm{ABC}$ analysis is a management method focused on the importance of a certain object as a share of the whole set of objects ${ }^{(2,6)}$. In the proposed method, we use a shipping quantity of each commodity for calculating the importance of a commodity among the whole set of commodities. Thus, the importance of a commodity is represented as the "quantity composition rate" of each commodity. This rate refers to the occupation rate of the shipping quantity of each commodity among a whole set of commodities. The actual procedure of $\mathrm{ABC}$ analysis in the proposed method follows four steps:

(a) The quantity composition ratio of each commodity was calculated using equation (1).

$$
O_{i}=\frac{A_{i}}{A_{t}} \times 100
$$

Where, $O_{i}$ is the quantity composition ratio of each commodity. The valuable $A_{i}$ is the sipping quantity of the commodity $i$, and $A_{t}$ is the total amount of whole commodities.

(b) Sort all the commodities in descending order based on the calculation result of the preceding process (a). After the sorting process, the cumulative value of the composition ratio at each rank was calculated using
Table 1. Commands used and the selection criteria.

\begin{tabular}{|c|c|c|c|c|c|}
\hline & $\begin{array}{c}\text { Sales } \\
\text { slip } \\
\text { number }\end{array}$ & $\begin{array}{c}\text { Customer } \\
\text { code }\end{array}$ & $\begin{array}{c}\text { Commodity } \\
\text { code }\end{array}$ & $\begin{array}{c}\text { Shipment } \\
\text { quantity }\end{array}$ & $\begin{array}{c}\text { Order } \\
\text { receipt date }\end{array}$ \\
\hline Order 1 & 358844 & YGNVU & Sm90 & 500 & 20XX0101 \\
\hline Order 2 & 358844 & YGNVU & Oj29 & 10000 & 20XX0101 \\
\hline Order 3 & 149060 & MRCGI & Xd87 & 10 & 20 XX0211 \\
\hline Order 4 & 052620 & XLWRU & Cq25 & 10 & $20 X X 0523$ \\
\hline Order 5 & 634711 & HHKTH & Hn21 & 90 & 20XX0729 \\
\hline
\end{tabular}

(a) Calculate the total amount of each commodity for all orders and quantity composition ratio for each commodity

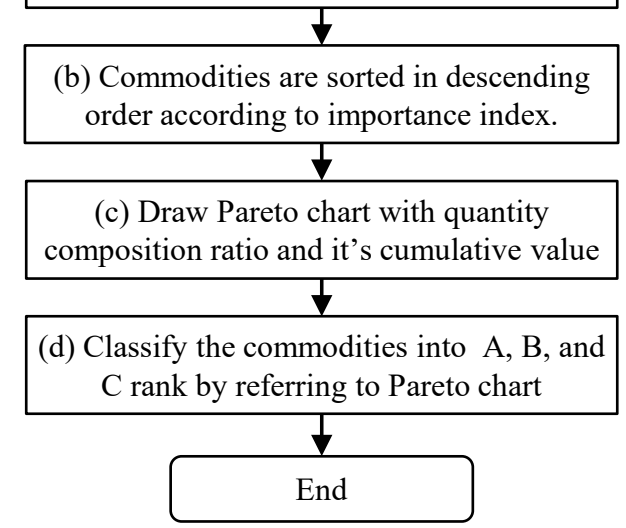

Fig. 4. Flow of $\mathrm{ABC}$ analysis.

equation (2)

$$
C O_{i}=\sum_{i=1}^{t} O_{i}
$$

Where, $\mathrm{CO}_{i}$ is the cumulative quantity of composition ratio of the commodity $i$, and $t$ is the total types of whole commodities. Table 2 shows an example of the processing result.

(c) The Pareto chart with the quantity composition ratio and cumulative value was made based on the result of process (b).

(d) All of the commodities are classified into three categories such as rank $\mathrm{A}, \mathrm{B}$, and $\mathrm{C}$ by referring to the Pareto chart. It is thought to be reasonable to mark off the boundary lines at cumulative composition rates of 60 to 85 percent, and at 90 to 95 percent, respectively. Therefore, we mark off the two boundary lines at the 90 
Table 2. An example of process (b) result.

\begin{tabular}{|c|c|r|r|}
\hline $\begin{array}{c}\text { Commodity } \\
\text { code }\end{array}$ & $\begin{array}{c}\text { Shipping } \\
\text { quantity }\end{array}$ & $\begin{array}{c}\text { Quantity } \\
\text { composition rate [\%] }\end{array}$ & $\begin{array}{c}\text { Cumulative value of } \\
\text { composition rate [\%] }\end{array}$ \\
\hline Hx92 & 317620 & 11.0 & 11.0 \\
\hline Lo35 & 287220 & 9.9 & 20.9 \\
\hline Wv68 & 245710 & 8.5 & 29.4 \\
\hline Nw94 & 234350 & 8.1 & 37.5 \\
\hline Ck84 & 162925 & 5.6 & 43.2 \\
\hline Oj29 & 162875 & 5.6 & 48.8 \\
\hline Iz20 & 94680 & 3.3 & 52.1 \\
\hline I & $\mid$ & & \\
\hline Xy05 & 30 & 0.0 & 100.0 \\
\hline
\end{tabular}

and 70 percent rates, respectively, in the proposed method.

\subsection{Determination of the Commodity Location}

The commodity location is determined by referring to the results of the ABC analysis. Fig. 5 shows an image of the commodity location using the proposed method. The determination process has three steps. First, preserve the number of racks required to store the commodities in each rank, and the preserved racks are allocated near the entrance with the calculated priority ranks (i.e., from the rank A to rank C). We used Manhattan distance to determine the proximity between the entrance and each rack. Next, commodities belonging to rank $\mathrm{A}$ are located at the preserved nearest racks, and commodities belonging to rank $\mathrm{B}$ are located at the preserved next nearest racks as shown in Fig. 5. In the areas for each rank, the commodity that has the greatest shipping quantity is located on the nearest rack in each rank area; after that, commodities are located on the next nearest racks in descending order based on shipping quantity. After the location process, overwrite the location records to the property of each commodity as shown in Table 3 . Then, the determination of the commodity location process is finished.

\section{Experiment}

\subsection{Outline of Experiment and Data Used}

To confirm the shortening effect of the proposed method for picker travel distance, we compared the picker travel distances between a location method that located commodities in the order listed in the original total picking

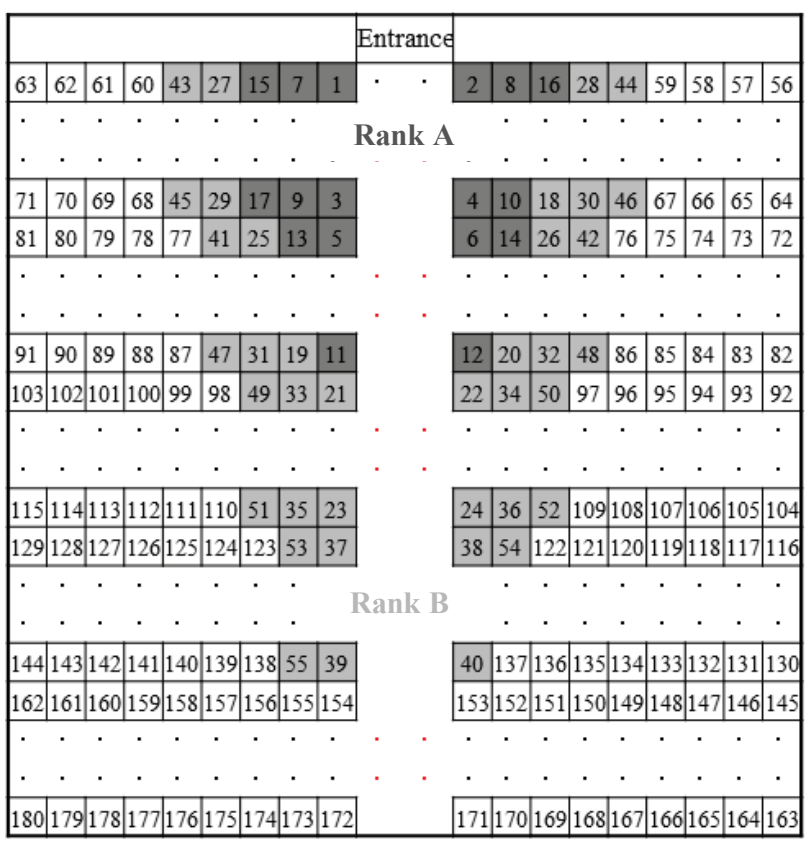

Fig. 5. An image of rack layout after relocation.

Table 3. An example of the commodity record after the location process.

\begin{tabular}{|c|c|c|c|}
\hline $\begin{array}{l}\text { Commodity } \\
\text { code }\end{array}$ & \begin{tabular}{|c|} 
Quantity \\
composition rate \\
{$[\%]$} \\
$\%]$
\end{tabular} & $\begin{array}{c}\text { Cumulative value } \\
\text { of composition } \\
\text { rate }[\%]\end{array}$ & $\begin{array}{c}\text { Rack number } \\
\text { (Relocate) }\end{array}$ \\
\hline Hx92 & 11.0 & 11.0 & 1 \\
\hline Lo35 & 9.9 & 20.9 & 2 \\
\hline Wv68 & 8.5 & 29.4 & 3 \\
\hline Nw94 & 8.1 & 37.5 & 4 \\
\hline Ck84 & 5.6 & 43.2 & 5 \\
\hline Oj29 & 5.6 & 48.8 & 6 \\
\hline Iz20 & 3.3 & 52.1 & 7 \\
\hline | & 1 & 1 & | \\
\hline Xy05 & 0.0 & 100.0 & 494 \\
\hline
\end{tabular}

slip and the proposed method. Fig. 6 shows the outline of this experiment.

Used data was seven months' actual business data provided from the Research and Development (R\&D) partner company. The number of original order receptions are 22,467, and 494 types of commodities are included in the data. Working days are 210 days for 7 months, and the average is 106 order receptions for a day. The commodity location number is the same as the rack number such as 1 to 494 for storing one type of commodity by one rack. The commodity size contains only one size. The outer dimension of the commodity size was set to length $40 \mathrm{~mm}$ $\times$ width $40 \mathrm{~mm} \times$ height $70 \mathrm{~mm}$. 


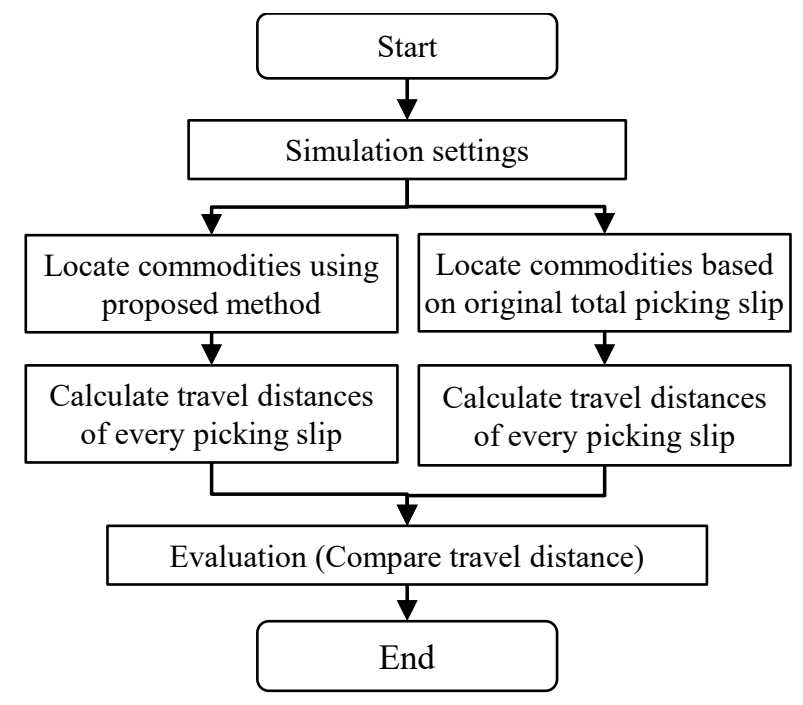

Fig. 6. Outline of experiment and comparison process.

\subsection{Warehouse Layout setting}

Fig. 7 shows the warehouse layout for this simulation. In a stock zone, 504 racks were set for storing 494 types of commodities. As already mentioned above, only one type of commodity was located on each rack, and the commodity location number was the same as the rack number. Based on the result of our field survey and general knowledge, racks were arranged at the left and right side of the center aisle symmetrically. The number of all of the racks used in this simulation had a single stage because our examination target is the travelling distance for total picking. The dimension of the rack size was width $1000 \mathrm{~mm} \times$ depth 600 $\mathrm{mm}$.

\subsection{Routing Method}

In this experiment, we applied a basic method for picker routing ${ }^{(2,3)}$. Fig. 8 shows an image of the routing method used in this simulation. The picker walks a U-shaped line along the right rack in one stroke writing manner.

In our warehouse model, the picker uses a general type of handcart for the manual picking operation, and the picker manages the picking work of one zone exclusively. Therefore, when an order item quantity exceeds the capacity of the handcart, it is necessary to unload the pick items at the depot. We define the unload process as one node named "RN: Return Node." The coordinates of the RN have the same coordinates as the entrance node, because the depot is set outside of the entrance. The capacity of the handcart was determined by the volume of the container that can be mounted on the handcart. The

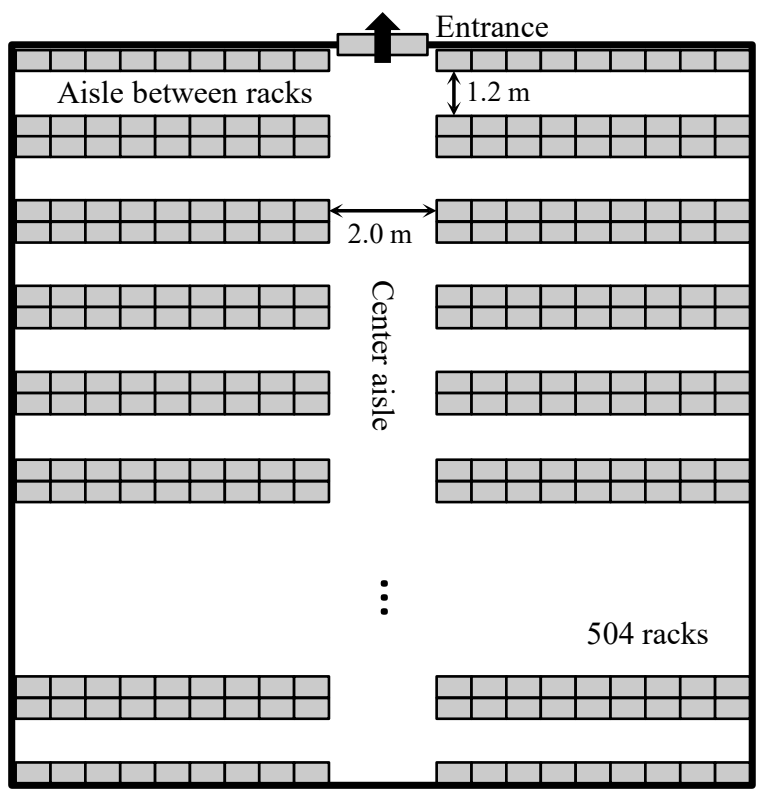

Fig. 7. Warehouse layout setting for experiment.

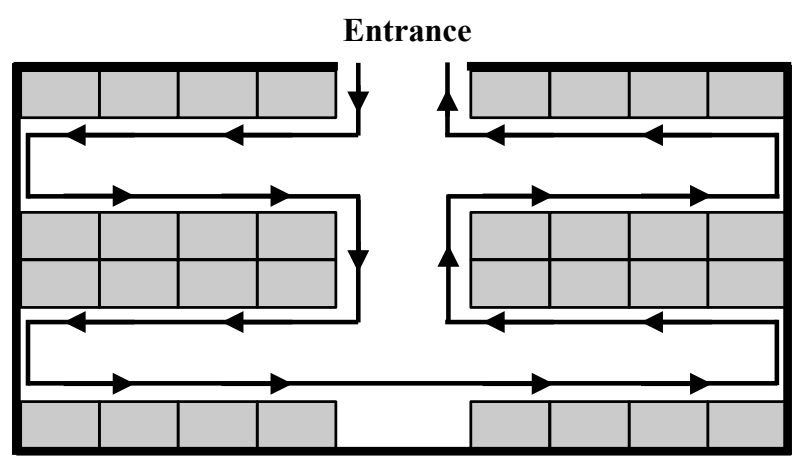

Fig. 8. Routing image.

container size was set to length $780 \mathrm{~mm} \times$ width $470 \mathrm{~mm} \times$ height $503 \mathrm{~mm}$ based on our survey about the general types of containers.

Fig. 9 shows the flowchart of the unloading process. The picked item quantity for one work period is counted. When the item quantity exceeds the capacity of the hand cart $\left(C_{N p} \geqq L_{m a x}\right)$, add one return node $\left(C_{R N}=C_{R N}+1\right)$. This process is repeated until the number of items for all nodes (racks) are picked $\left(N_{p}=N_{l}\right)$.

\subsection{Experimental Result}

Fig. 10 shows the Pareto chart calculated by the proposed method. The top 17 commodities occupied approximately 70 percent of the entire shipping quantity, and occupancy was increased up to approximately 90 percent by adding the next 38 commodities. Thus, the top 17 commodities were set to rank $\mathrm{A}$, the next 38 commodities were set to rank $\mathrm{B}$, and the other 439 


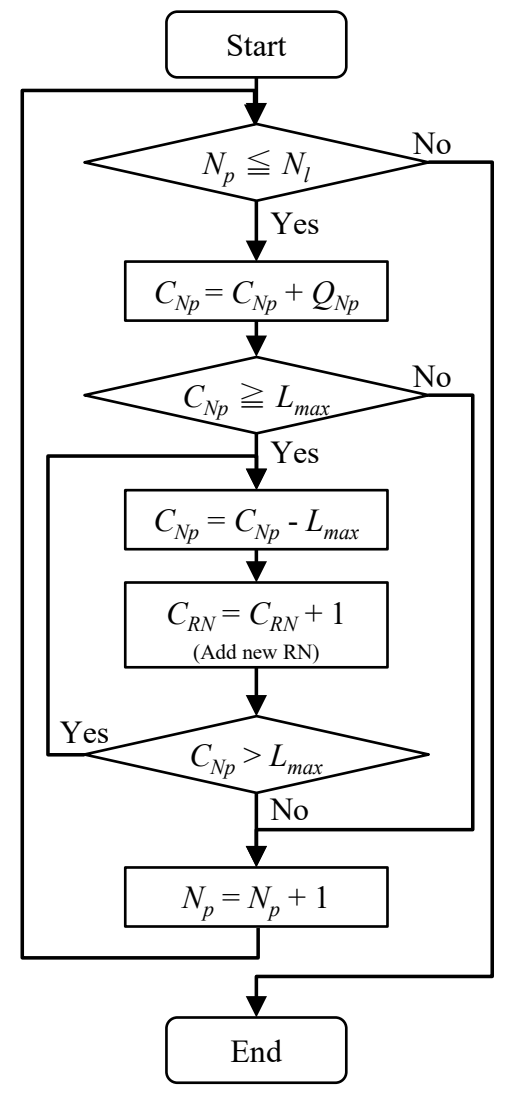

$N_{p}$ : Present travelling node

$Q_{N p}$ : Quantity of load items at $N_{p}$

$L_{\max }$ : Maximum capacity of using hand cart

$C_{N p}$ : Counter valuable for cart loading at $N_{p}$

$C_{R N}$ : Counter valuable for the number of $\mathrm{RN}$

$N_{l}$ : The last node for a picking work

Fig. 9. Flow chart of unloading process.

commodities were set to rank C. Commodities were located on the basis of the classification.

Table 4 compares the results of both methods. It is reveals that travel distance is decreased drastically by use of the proposed method; this is $45.8 \%$ shorter than that of the original location (not changed location) in the mean value. The proposed method decreases the travel distance in all months; the shortening rate of each month ranges from $44.0 \%$ to $47.8 \%$. There are various factors behind this result, one of which is the difference in travel distance on the center aisle, because the simulation model in this experiment has a loading process. The picker returns to the depot through the center aisle for unloading the commodities when the container on the handcart is filled with commodities. The loading process tends to occur frequently at a rack that has the largest shipping order amount. The proposed method locates a large quantity of commodities near the entrance. Therefore, it is thought that the return distance of the changed location by the proposed

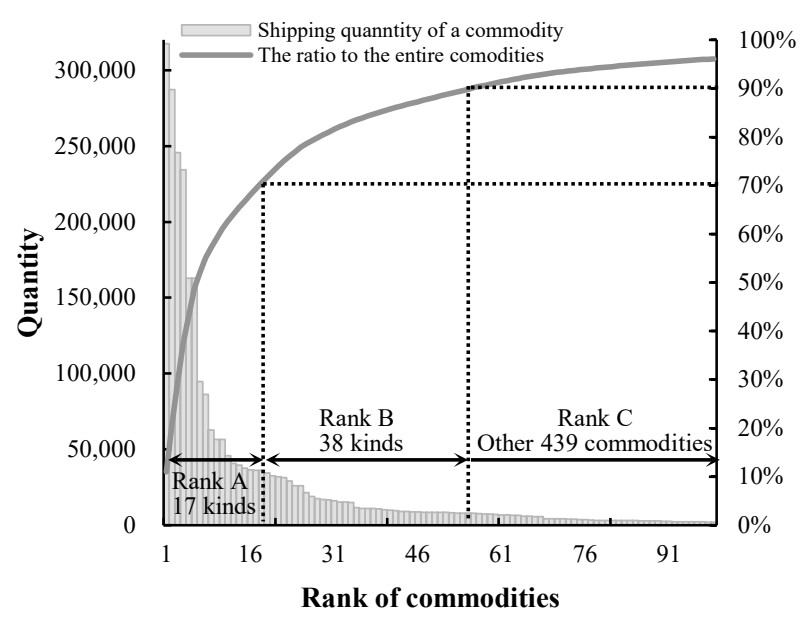

Fig. 10. Pareto chart of data used.

Table 4. Compared results of the both methods.

\begin{tabular}{|l|c|c|r|}
\hline Month & $\begin{array}{c}\text { Original } \\
\text { location } \\
{[\mathrm{m}]}\end{array}$ & $\begin{array}{c}\text { Changed } \\
\text { location } \\
{[\mathrm{m}]}\end{array}$ & $\begin{array}{c}\text { Shortening rate between } \\
\text { the original location and } \\
\text { the changed location }\end{array}$ \\
\hline January & 37492.1 & 21005.8 & $44.0 \%$ \\
\hline February & 39170.7 & 21400.3 & $45.4 \%$ \\
\hline March & 44298.8 & 23735.4 & $46.4 \%$ \\
\hline April & 47332.2 & 24726.4 & $47.8 \%$ \\
\hline May & 38758.6 & 21068.7 & $45.6 \%$ \\
\hline June & 43706.8 & 23687.3 & $45.8 \%$ \\
\hline July & 41827.5 & 22865.5 & $45.3 \%$ \\
\hline Average & 41798.1 & 22641.3 & $45.8 \%$ \\
\hline
\end{tabular}

method is shorter than that of the original location. Further investigation will be needed to clearly identify other factors.

In addition, the traveling distances of April and March, respectively, were longer than other months, and those two months generated high shortening rates as well. In contrast, the traveling distance of January generated a lower shortening rate. From these results, it is thought that the shortening rate tends to rise as the traveling distance of the original location increases.

\section{Conclusions}

In this study, we developed the commodity location method for the picking support system used in medium or small-sized warehouses. The proposed location method uses $\mathrm{ABC}$ analysis to determine the relative importance of shipping commodities. To investigate the effect of the 
changed locations, we compare the travel distance between the changed location due to the commodity location method and the original location. Our experiments provide the following conclusions:

- Experiment results reveal that the travel distance was decreased drastically by use of the proposed method; it was $45.8 \%$ shorter in mean value than that of the original location.

- Experimental results indicate a tendency for the shortening rate of the travel distance becomes more effective as the travel distance of the original location increases.

For future work, we plan to investigate the other factors for shortening travel distance and increasing the effectiveness of the proposed location method. In addition, we also plan to develop a picking support system with a travel route search method adapted to a medium or smallsized warehouse.

\section{Acknowledgments}

The authors would like to thank Dr. C. Ishizawa, Akita University, Japan, for her assistance with the experiments; and Mr. Kenta Suzuki Department of Computer Science and Engineering, Akita University, Japan, for his assistance in this study. In addition, the authors would like to thank Best Co., Ltd. and Best Sewing Co., Ltd. for their cooperation on this study.

\section{References}

(1) R. De Koster, T. Le-Duc, K. J. Roodbergen : "Design and Control of Warehouse Order Picking: A Literature Review", European Journal of Operation Research, Vol. 182, Issue 2, pp. 481-501, 2007

(2) K. Namikata: "A New Common Sense Approach to Logistics ”, PHP Institute, Inc., 2008 (in Japanese)

(3) Y. Sato: "Butsuryu, Haisou Center Setti to Kourituka no Point (The Improvement on the Distribution Center)", Nikkan Kogyo Shimbun Ltd., 1993 (in Japanese)

(4) A. Kunisada: "Scientific Approach to Improvement of Logistics Business", UNISYS TECHNOLOGY REVEW, Vol. 59, pp. 134-150, 1998 (in Japanese)

(5) J. P. van den Berg and W. H. M. Zing: "Models for Warehouse Management: Classification and Examples ", International Journal of Production
Economics, Vol. 59, pp. 519-528, 1999

(6) T. Okamoto, T. Sakurai: "Korede Anshin Shigoto no Kihon ga Yoku Wakaru Zaiko Kanri no Shikumi to Jitsumu (The structure and business of inventory management)", Dobunkan Publishing Co., Ltd., 2000 (in Japanese) 